\title{
Multi-Dimensional Laser Confocal Microscopy on Live Cells in Submicroliter Volumes Using Glass Capillaries
}

\author{
Emilie Flaberg$^{1}$, György Stuber ${ }^{1}$ and Laszlo Szekely ${ }^{1}$ \\ ${ }^{1}$ Microbiology and Tumor Biology Center (MTC) and Center for Integrative Recognition in the Immune System (IRIS), \\ Karolinska Institute, S-17177 Stockholm, Sweden
}

Received December 26, 2005; accepted April 25, 2006; published online June 2, 2006

\begin{abstract}
Imaging live cells using laser confocal microscopy requires the use of complex and rather cumbersome incubation chamber systems in order to maintain the correct physiological conditions. The volume of these chambers is in the range of a few hundred microliters. Here we present an easy and convenient alternative in the form of glass capillaries that accommodate volumes of $0.2-10$ microliters. The capillaries can be loaded with both suspension and adherent cells. The loaded capillaries are taped on microscope slides and submerged into the immersion oil that covers the objective. The correct temperature is maintained using a thermostat-controlled objective heater. We demonstrate that using microlens enhanced rotating Nipkow disc based confocal illumination, in combination with cold CCD cameras, maximum resolution multicolor time lapse fluorescence images can be obtained from live cells. The images obtained are free from disturbing optical distortions. Imaging in submicroliter volumes allows for fluorescence visualization of very rare cell types isolated using flow or affinity sorting or obtained by fine needle biopsies.
\end{abstract}

Key words: capillary, live cell imaging, confocal, spinning disc, fluorescence

Introduction of microlens enhanced rotating Nipkow spinning disc confocal illumination in combination with cold CCD camera based image acquisition, opened new perspectives in fluorescence live cell imaging [2]. Conventional laser scanning confocal microscopy is often too slow and insensitive for many live cell imaging applications. It also exposes the cells to dangerously high doses of photons that can lead to light induced $\mathrm{Ca}^{+}$signaling mediated apoptosis [4]. The greatly reduced phototoxicity using spinning disc based parallel beam illumination systems allows threedimensional multicolor time-lapse imaging over several hours.

Keeping cells alive for extended periods of time requires careful control of temperature, $\mathrm{pH}$ and supply of nutrients [3]. There are several ways to create correct incubation conditions. Live cell imaging using open tissue culture dishes requires the installation of a large incubation

Correspondence to: Laszlo Szekely, Microbiology and Tumor Biology Center (MTC) and Center for Integrative Recognition in the Immune System (IRIS), Karolinska Institute, S-17177 Stockholm, Sweden. E-mail: lassze@ki.se chamber that surrounds half of the microscope. It also requires elaborate machinery to provide correct temperature and humidity as well as correct $\mathrm{CO}_{2}$ partial pressure. A more convenient live cell incubation device is exemplified by so called POC mini chambers $[1,6]$. These chambers consist of a metal base plate with high heat capacity that accommodate $30 \mathrm{~mm}$ diameter coverslips that sit in a round opening. Two coverslips separated by a silicon ring enclose the living cells in the tissue culture medium. The smallest possible fluid volume that is needed to fill the chamber is $400 \mu \mathrm{l}$. The POC mini chambers can be operated in open, closed or perfusion modes. Open mode operation requires the use of the above mentioned large incubation chamber. When the POC mini chamber is used in closed or perfusion modes, the temperature is controlled by a thermostat-regulated heating stage that is mounted on the object table. To prevent extensive heat loss toward the objective this table has to be equipped with a thermostat-controlled heater ring. This state of the art assembly still has a few shortcomings. The system is relatively expensive. It does not work with small volumes. It cannot be used for parallel imaging of multiple samples.

With modern cell separation techniques came the pos- 

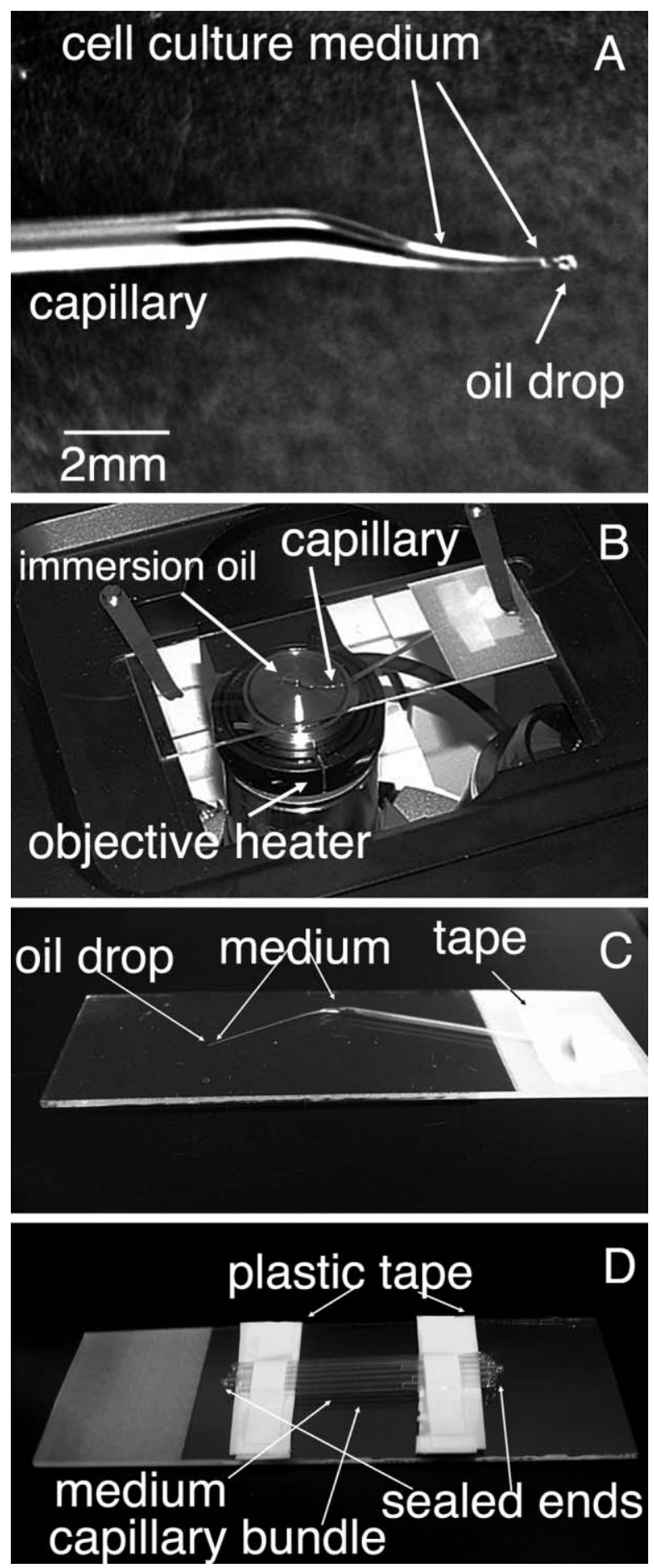

Fig. 1. Glass capillary set-ups for live cell imaging. Glass capillary loaded with cell culture medium and sealed with oil drop (A). Positioning of glass capillary on an inverted oil immersion objective equipped with an external heating ring (B). Hair thin capillary mounted on glass slide (C). Permanently sealed capillary bundle mounted on glass slide for automatic parallel imaging of cells in small volumes (D). sibility to analyze small volumes with only a few number of cells. Flow sorting or magnetic bead binding techniques allow isolation of homogeneous populations of rare cells from complex sources such as blood, bone marrow, spleen or collagenase dissociated solid organs. Typical examples are stem cells, rare immunological effector cells, minor populations of infiltrating tumor cells or virus infected cells. Additionally, cells from very small samples such as needle biopsies, embryos or tissue samples from small experimental animals also need to be analyzed in submicroliter volumes.

We therefore developed a technique for live cell imaging in submicroliter volumes. We reasoned that, by using immersion oil with the same refractory index as the wall of the incubation vessel, the geometry of the wall does not necessarily have to be on a parallel plane as long as we use spinning disc confocal technology with a thousand parallel beams for every illuminated area. We tested two types of glass capillary set-ups. In both cases we used $10 \mu \mathrm{l}$ glass capillaries (Idaho Technology Part \#1705) designed for capillary PCR.

In the first set-up we loaded the capillaries with $2-4 \mu \mathrm{l}$ cell suspensions by immersing one end of the glass tube into the cell culture fluid drop sitting on parafilm. The capillaries were sealed at both ends with standard butane burners. The sealed capillaries were taped on the surface of glass slides. To avoid heat loss toward the glass slide two slices of a four layer thick plastic electric isolation tape strip were wedged between the capillaries and the slide. The slides were placed on a Zeiss Axiovert inverted fluorescence microscope where the objective was equipped with a thermostat-regulated heating ring. Suspension cells that were gathered on the bottom of the capillaries were conveniently imaged using $16 \times, 40 \times$, $63 \times$ or $100 \times$ oil immersion objectives using laser confocal illumination with CSU10 or CSU21 microlens enhanced rotating Nipkow disc units (UltraView LCI or UltraView RS-5 live cell imaging systems, both from Perkin-Elmer). Argon/krypton (488, 568, $647 \mathrm{~nm})$, Argon ion (488, $514 \mathrm{~nm})$ and Krypton (568 nm) gas lasers as well as 405 and $640 \mathrm{~nm}$ solid state lasers were used as illumination sources. The images were recorded using Hamamatsu Orca ER cold CCD cameras. No disturbing optical distortion was detectable. Cells expressing recombinant EGFP, YFP or CFP fusion proteins were routinely examined over several hours using multicolor plus 4D (optical sectioning with time lapse) imaging. The cellular DNA was visualized using the infrared emitting live cell permeable dye DRAQ5 [5]. The advantage of this set-up is that because the capillaries are firmly sealed there is no danger of evaporation of the medium. The cells can be kept alive in the capillaries over $24-48$ hours. The sealed capillaries are sturdy and can be conveniently sterilized by immersing them in formalin. This method even allows live cell imaging of infectious agents. Moreover the uniformly sealed capillaries can be mounted as a parallel bundle on the same glass slide. This arrangement allows parallel imaging of multiple sites if the microscope is equipped with a computer controlled XY stage. Sequential, 


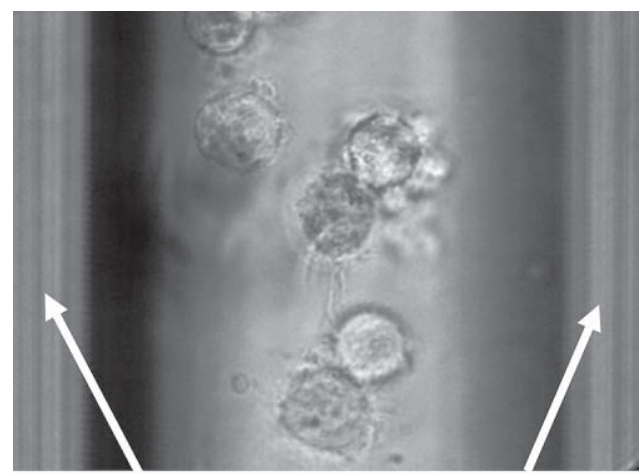

\section{walls of the capillary}

Fig. 2. Living EBV immortalized human B-cells in the lumen of a hair thin capillary visualized by transmitted light.

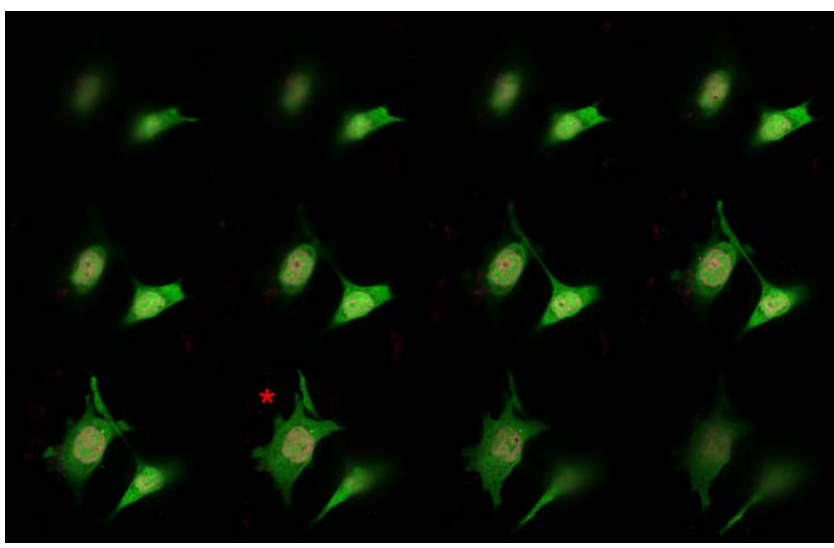

Fig. 3. Confocal optical sectioning of living, adherent human osterosarcoma cells, Saos-2, grown on the inner surface of a hair thin glass capillary. The cells constitutively express EGFP. Nucleus is stained by the live cell permeable fluorescent dye DRAQ5. The cell marked by the red star $(*)$ is shown in high resolution on the next figures.

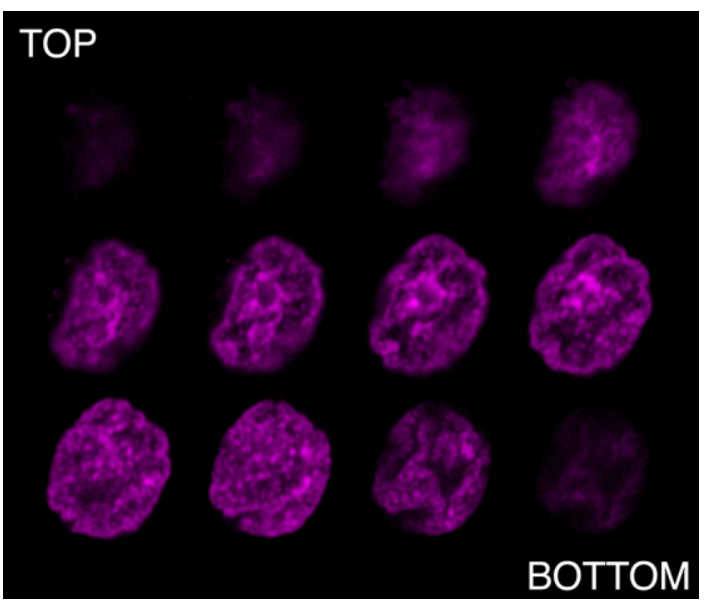

Fig. 4. Confocal optical sectioning of the nucleus of a living Saos-2 cell (* labeled on the previous figure), grown on the inner surface of a hair thin capillary, demonstrates distortion free imaging at high resolution (63× NA $1.25 \mathrm{Ph}$ objective). The DNA is stained with DRAQ5.

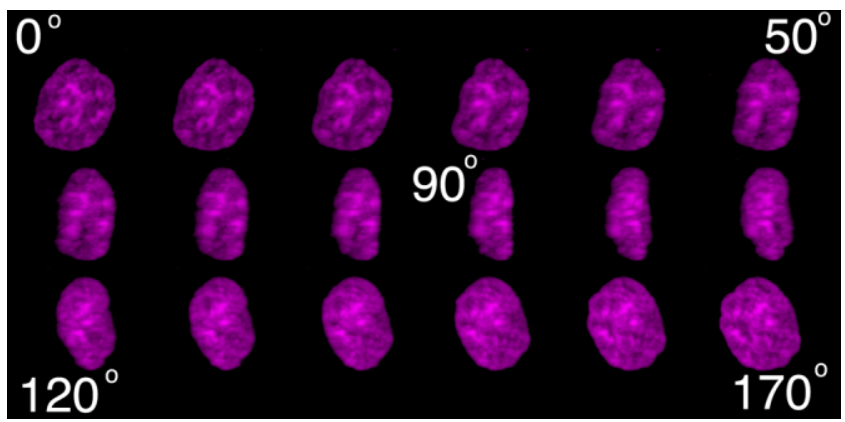

Fig. 5. Three dimensional reconstitution of the nucleus of the living Saos-2 cell from Figure 4. Half circle rotation along the $\mathrm{Y}$-axis in 10 degree increments.

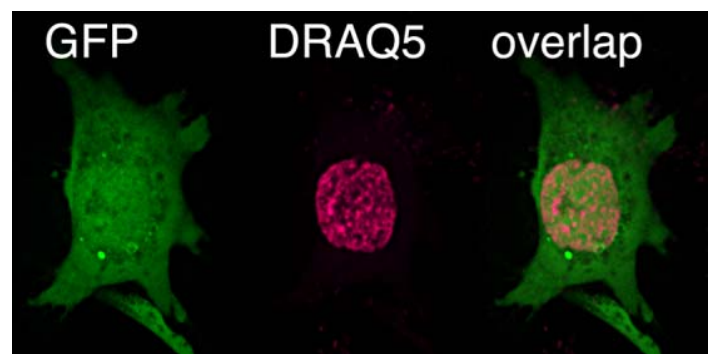

Fig. 6. Single optical section of the living Saos-2 cell expressing EGFP, growing on the inner surface of a hair thin capillary. The nucleus is stained with DRAQ5.

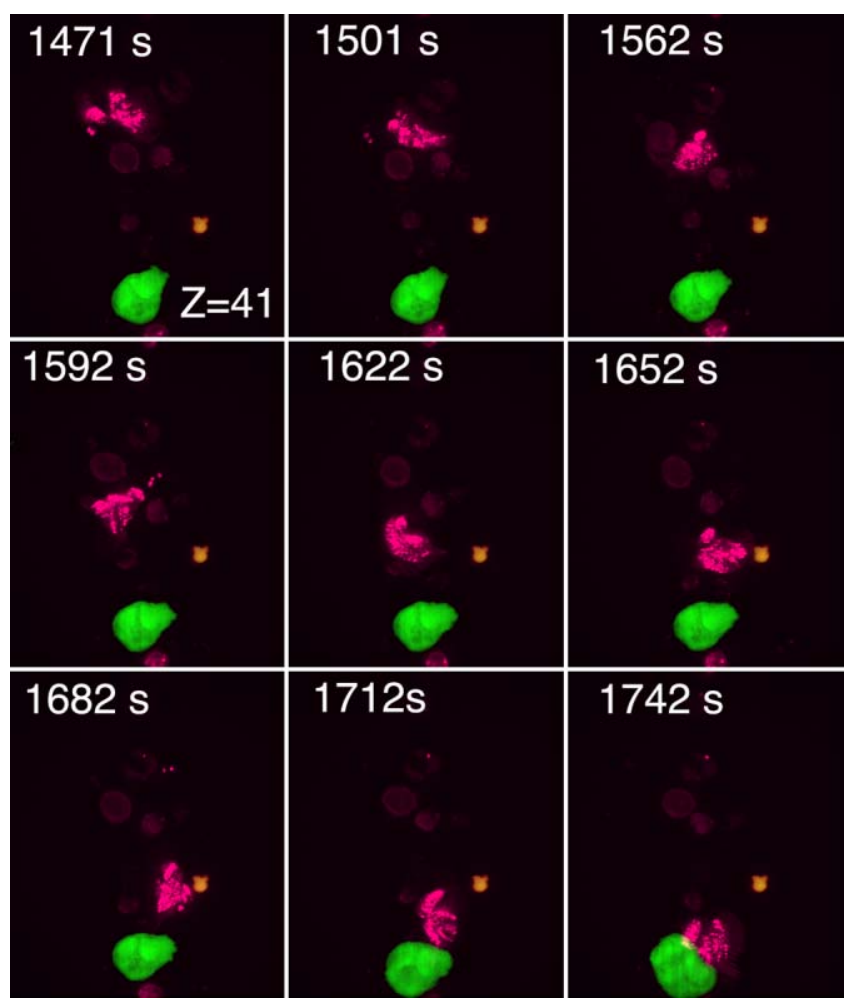

Fig. 7. A 4.5 minute excerpt from a 45 minute 5 dimensional confocal time-lapse recording depicting the engagement of a human NK cell (magenta dots in the cytoplasm) and a recombinant GFP carrying Epstein-Barr virus infected Burkitt lymphoma (Akata) cell. Each picture is a projection of 41 confocal sections 0.5 micrometers apart. 
recursive time lapse imaging of multiple sites can be conveniently carried out using programmable microscope control software such as ISee (ISIS) or Openlab Automator (Improvision).

To image monolayer cells the capillaries can be first loaded with trypsinized cells. The loaded unsealed capillaries are incubated fully submersed in medium (in Petri dishes) to allow the monolayer to be formed on the inner wall of the capillary. Before sealing the capillaries, the medium can be changed by ejecting the used medium with the help of a $2 \mathrm{ml}$ syringe and a $2 \mathrm{~mm}$ thick polypropylene chromatography tube. Subsequently a new medium is loaded by capillary action that contains the appropriate fluorescence dyes, biological effector molecules and/or interacting cells or microbes.

In the second set-up we intended to further decrease the incubation volume. We pulled capillaries hair thin by melting the middle of the $10 \mu \mathrm{l}$ glass capillary with a butane burner. The glass capillaries were perpendicularly suspended on a test tube holder stage with a metal clip under the weight of another clip (18 gr). Slowly melting the middle section with the edge of the flame led to the formation of very thin capillaries with 50-150 $\mu \mathrm{m}$ inner diameter and 25 $50 \mu \mathrm{m}$ wall thickness. The thin section was broken with scissors, $3 \mathrm{~cm}$ after the molten shoulder. The hair thin capillaries were also loaded by capillary action. A microliter-size drop of cell suspension was placed on parafilm and the thin end of the capillary was touched to the drop. When $0.2-0.5 \mu \mathrm{l}$ was loaded, the thin end was sealed by briefly touching a small drop of PCR grade paraffin oil. The thick end of the capillary was sealed with water saturated gummi arabicum. The sealed capillary was taped on a glass slide in such a way that the hair thin section was suspended $1 \mathrm{~mm}$ below and parallel to the glass slide. The hair thin section of the capillary was submersed in the warm immersion oil sitting on the top of the heated objective (Fig. 1). By this method a few dozen cells are sufficient to carry out multidimensional live cell imaging (Figs. 2-6). Our method can be particularly suitable for studying the interactions between immunological effec- tor and target cells where the number of available cells are limited (Fig. 7). To conveniently spatially orchestrate the interactions the hair thin capillary can be sequentially loaded first with the target and then with the effector cells. The border between the two volumes constitutes the high likelihood surface of interactions. This second set-up, however, is somewhat fragile. Due to the lack of permanent glass sealing it is not recommended for work with high-risk human pathogens. On the other hand it may be particularly suitable to study very rare cells or cells that are treated with biological agents that are very expensive or difficult to obtain.

Live cell imaging in glass capillaries may allow functional studies on primary cells that are obtained using fine needle biopsy of tumors or different normal or diseased organs.

\section{Acknowledgment}

We want to thank Victor Levitsky and Noemi Nagy for providing the NK cells and GFP-EBV infected Akata cells as well as the EGFP-expressing Saos-2 cells, respectively.

\section{References}

1. Freimann, R., Pentz, S. and Horler, H. (1997) Development of a standing-wave fluorescence microscope with high nodal plane flatness. J. Microsc. 187; 193-200.

2. Graf, R., Rietdorf, J. and Zimmermann, T. (2005) Live cell spinning disk microscopy. Adv. Biochem. Eng. Biotechnol. 95; 57-75.

3. Gura, T. (1997) Biologists get up close and personal with live cells. Science 276: 1988-1990.

4. Knight, M. M., Roberts, S. R., Lee, D. A. and Bader, D. L. (2003) Live cell imaging using confocal microscopy induces intracellular calcium transients and cell death. Am. J. Physiol. Cell Physiol. 284; C1083-C1089.

5. Martin, R. M., Leonhardt, H. and Cardoso, M. C. (2005) DNA labeling in living cells. Cytometry A. 67; 45-52.

6. Pentz, S. and Horler, H. (1992) A variable cell culture chamber for 'open' and 'closed' cultivation, perfusion and high microscopic resolution of living cells. J. Microsc. 167; 97-103. 